\title{
Sensors Based on Tunnel Magnetoresistance - New Technology, New Opportunities
}

\author{
Johannes Paul ${ }^{1}$, Christian Schnieders ${ }^{1}$, Jörg Traute $^{1}$, Ronald Lehndorff ${ }^{1}$, Andrés Conca ${ }^{2}$, \\ Frederick Casper ${ }^{3}$, Gerhard Jakob ${ }^{3}$, Mathias Kläui ${ }^{3}$, Britta Leven ${ }^{2}$, Burkhard Hillebrands $^{2}$, \\ ${ }^{1}$ Sensitec GmbH, Hechtsheimer Straße 2, 55131 Mainz, Germany, \\ Ronald.Lehndorff@sensitec.com \\ ${ }^{2}$ Fachbereich Physik and Landesforschungszentrum OPTIMAS, Technische Universität \\ Kaiserslautern, Germany \\ ${ }^{3}$ Institut für Physik, Johannes Gutenberg - Universität Mainz, Germany
}

\begin{abstract}
:
Magnetoresistive sensors based on the tunnel magnetoresistance effect penetrate more and more the sensor market. They provide high signal amplitude which enables the use of microcontrollers or ASICs without expensive preamplifiers. In addition high-impedance sensors can be designed with little chip area consumption. The combination of these two properties makes TMR sensors ideally suited for battery powered applications. Reliability issues, mainly the long term stability of the ultrathin barrier layer are solved. Angle and position sensors have been established. They reach an absolute accuracy which is close to that of AMR sensors.
\end{abstract}

Key words: tunnel magnetoresistance, angle sensor, position sensor, reliability, MR

\section{Why TMR?}

A wide range of magnetic sensors is available today. High volume market is dominated by Hall sensors, mainly because they are monolithically integrated into ASICs providing very cheap and small solutions. For applications with high resolution or high temperature requirements, magnetoresistive sensors are regarded as a better alternative to Hall sensors. Here the market is still dominated by AMR (anisotropic magnetoresistive) sensors, which are very simple, extremely robust, and able e.g. to

Magnetic tunneling junction

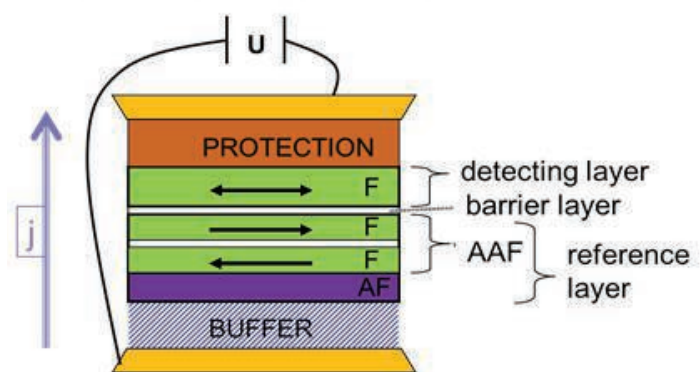

Figure 1: Schematic drawing of a TMR spinvalve structure. $F$ stands for ferromagnetic, AF for antiferromagnetic and AAF for artificial antiferromagnet. Current flows perpendicularly to the layer structure detect the angle of a magnetic field with an absolute accuracy better than $0.1^{\circ}$ [1]. GMR (giant magnetoresistive) sensors entered the market in the last decade, too. They offer larger amplitude and smaller size compared to AMR but not better resolution. The monolithic integration of magnetoresistive sensors exists but is not standard. It is realized typically by piggyback design: the MR part is deposited on top of the ASIC, normally in a manufacturing line which is separated from the semiconductor fab. Knowing the plurality of magnetic sensors, it is evident that a new technology can only be successful in the market if it convinces with unique features and advantages compared to existing solutions.

\section{Functionality of TMR Sensors}

TMR sensors are based on the spinvalve design, having a magnetic reference layer, for which the direction of magnetization is fixed or pinned, and a magnetic sensing layer, where the magnetization follows easily the external field (see Fig. 1). Between the reference and the sensing layer there is a thin isolating barrier, typically made from $\mathrm{MgO}$ or $\mathrm{Al}_{2} \mathrm{O}_{3}$. The barrier layer is thin enough for electrons to pass the isolation by tunneling. The resistance of such a tunnel junction is described by the formula

$$
R(\vartheta)=R_{0}-\Delta R \cos (\vartheta)
$$


where $\vartheta$ is the angle between the magnetization directions of reference and sensing layer and $R_{0}$ is the area resistance $R A$ divided by the area of the tunnel junction. $R A$ increases with the thickness $d$ of the barrier by an exponential function

$$
R A \sim e^{d / d 0}
$$

showing that the resistance of a TMR sensor is highly sensitive to the thickness of the barrier. The TMR effect is described by

$$
T M R=\frac{R_{\max }-R_{\min }}{R_{\min }} \cdot 100 \%
$$

$R_{\max }$ is the resistance in antiparallel orientation of the magnetization directions $\left(\vartheta=180^{\circ}\right)$ and $R_{\min }$ is the resistance in parallel configuration $\left(\vartheta=0^{\circ}\right)$. TMR effects as large as $200 \%$ can be achieved for junctions deposited in industrial production tools [2].

\section{Advantages of TMR Technology}

\section{High Resistance - Low Power Consumption}

TMR sensors cover a resistance range from a few Ohm to MOhm. It is possible to build high resistance and low resistance sensors with the same design just by changing the barrier thickness in the TMR deposition process. This approach needs no additional chip area in contrast to AMR or GMR technology where high resistance sensors have to be designed by endless long meanders. The area-powerproduct Vresource describes well this advantage of TMR:

$$
\text { Vresources }=A * P
$$

Vresource is a useful parameter which describes the consumption of resources of the sensing application $[3,4]$. $A$ is the area of the chip and $P$ is the required power consumption. Vresource is very low for TMR technology showing the high potential in mobile applications which are battery powered.

\section{Large Signal Amplitude}

The TMR effect is very high compared to other MR technologies. Therefore the output signal or sensitivity of a sensor is much larger. However, because of the much lower noise of AMR sensors compared to TMR sensors, there might be no significant advantage for detecting low signals $[5,6]$. True advantage is the fact that preamplifiers are not anymore strictly required when using TMR sensors. This shrinks the size and complexity of a PCB or of an ASIC and reduces the overall costs. In addition reducing the number of components in a measurement system increases the robustness of the system and facilitates failure analysis.

\section{Small Sensing Elements}

TMR junctions have lengths of typically a few micrometers and are nicely scalable. Sensing elements can be designed very small. Therefore it is possible to measure with high spatial resolution. This is important for applications like nondestructive testing or other imaging technologies [7]. It can also be used in length sensors or pole ring sensors with ultralow pitches.

\section{Risks of TMR Technology}

Beside the outstanding advantages there are some issues which must be taken into account.

\section{Critical Resistance Specification}

The exponential increase of the resistance with the barrier thickness allows the realization of low and high resistive sensors. On the other side it becomes a challenge to keep the resistance of the sensor constant during production. Tiny changes in MgO thickness immediately result in significant changes in resistance values. The barrier thickness should be controlled better than a tenth of a monolayer of $\mathrm{MgO}$ [8]. To achieve this precision a deposition tool with high uniformity and good repeatability is needed. Otherwise the final resistance specification of the sensor product has to be enlarged.

\section{High Investment}

TMR layer stacks consist of a higher number of individual layers compared to AMR sensing layers. At least five different materials are used in a TMR layer stack, e.g. Ru, Ta, CoFeB, $\mathrm{MgO}, \mathrm{PtMn}$, whereas AMR only needs two layers (e.g. NiFe and Capping). The $\mathrm{MgO}$ process should be improved by adding oxygen gas to the chamber which is not helpful for the metal layers at all. In addition there are high requirements on the uniformity of the deposited layers. Therefore a state of the art deposition tool with a minimum of five targets and two separated chambers (reactive and non-reactive deposition) is required to deposit TMR layer stacks.

In addition new testing tools for quality control are necessary. A standard 4-point probe to characterize the sheet resistance of a deposited layer is no more applicable to TMR layer stack. The needles will penetrate the film and damage the barrier immediately. To overcome this problem, a CIPT $^{1}$ tool is required [9]. Here, the

\footnotetext{
${ }^{1}$ CIPT stands for Current In Plane Test
} 
surface of the stack is contacted with ultrafine needle tips, similar as for scanning tunneling microscopes. No damage occurs at the layer surface.

\section{Possibility of Electric Breakthrough of the Barrier Layer}

The ultrathin barrier layer is highly sensitive to electrostatic discharges which will cause an electric shortcut. This problem can be prevented by adding ESD protection structures to the sensor. Much more fundamental is the electrical stress which acts permanently on the barrier. Investigations on the long term robustness of the barrier are crucial to design reliable sensors.

\section{Reliability of MgO Barrier}

Since the electrodes are metallic and the current is flowing perpendicular to the layers, the voltage drop is occurring over only a few nanometers at the barrier. The result is a high electric field in the order of $E=10^{6} \mathrm{~V} / \mathrm{cm}$. This electric field represents a stress agent which is able, after a certain time, to produce a dielectric breakdown of the barrier.

\section{Weibull Model for Thin Oxide Barriers}

A study of the breakdown processes in MTJs can only be done in a statistical way. From the investigations on $\mathrm{SiO}_{2}$ thin layers, it was first known that the dielectric breakdown can be described using Weibull statistics [10]. This was later confirmed for MTJs with $\mathrm{Al}_{2} \mathrm{O}_{3}[11,12]$ and $\mathrm{MgO}$ [13] barriers. The Weibull cumulative function describes the total fraction $F$ of broken elements after a certain time $t$.

$$
F(t)=1-\exp \left(-\left[\frac{t}{\eta}\right]^{\beta}\right)
$$

Here, it is straightforward to see that after a time $t=\eta, 63.2 \%$ of the junctions are broken. In order to understand the role of the shape

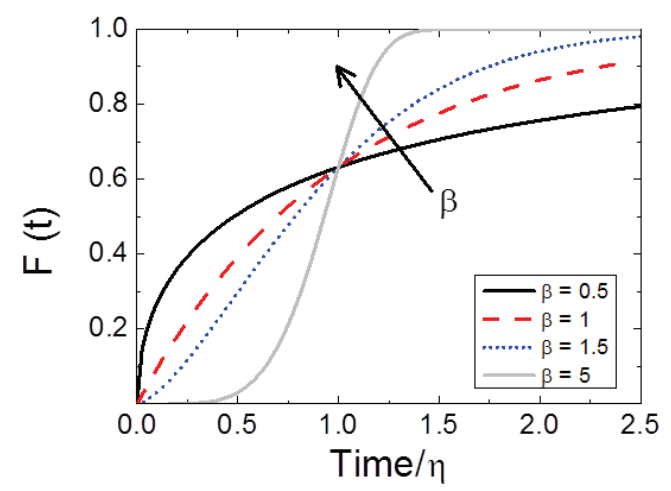

Figure 2: Weibull cumulative function $F(t)$ describing the total fraction of broken elements after a time $t$, calculated for different values of the shape parameter $\beta$

parameter $\beta, F(t)$ is plotted in Fig. 2 for several $\beta$ values. One can distinguish three cases. For $\beta<1$ (black line) a large infant mortality is present, i.e. a significant fraction of the elements breaks fast just after applying the stress. The opposite happens for $\beta>1$ (dotted data and grey line) where few elements break at the beginning and the failure rate increases with time (aging). The limit case $\beta=1$ implies a constant failure rate indicating a random external agent.

\section{Lifetime Dependence on MgO Thickness}

The characterization of the breakdown processes was applied for circular $\mathrm{CoFeB} / \mathrm{MgO} / \mathrm{CoFeB}$ junctions at applied voltages between 1.5 and $2.3 \mathrm{~V}$. Typical working condition voltages for MTJs are much lower than the voltages used here. However, the lifetimes in the low voltage regime can reach values well over $10^{6}$ years. It is impossible to get sufficient statistics with a reasonable measurement time at low voltages. For this reason the stress processes are accelerated by measuring at larger voltages. The measured lifetimes have been extrapolated to expected lifetimes at working conditions by means of a simple exponential law [14]

$$
\eta=A \exp (-B V) \text {. }
$$

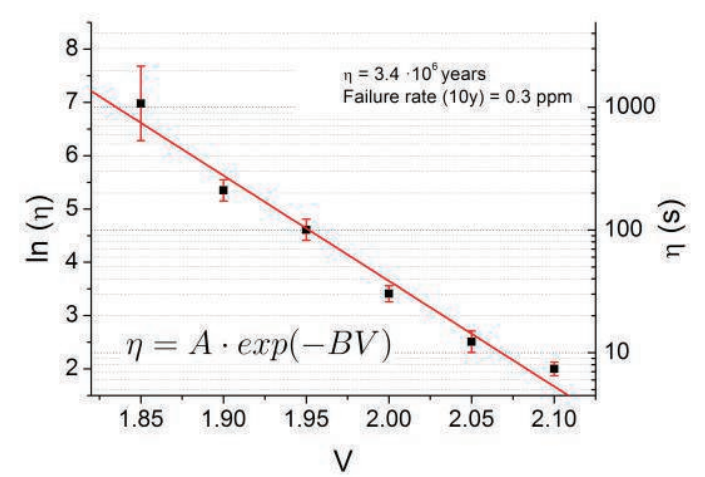

Figure 3: Dependence of the characteristic lifetime $\eta$ and $\ln (\eta)$ of tunneling junctions on the stress voltage.

Fig. 3 shows exemplarily the measured dependence of $\eta$ on the applied voltages. The line represents $a$ fit to the exponential law, which is able to describe the dependence. Now it is possible to extrapolate the lifetime for the low voltage regime $(0.4 \mathrm{~V})$. It is clear that the obtained value will be accompanied by a certain error bar. Since we are interested to evaluate a minimum guaranteed lifetime, a conservative approach recommends the use of the lowest possible value compatible with our measurement uncertainty (worst case 
scenario). This is, for the presented set of data $\eta(0.4 \mathrm{~V})=3.4 \cdot 10^{6}$ years which corresponds to a failure rate after 10 years of $0.3 \mathrm{ppm}$.

These results enable us to design sensors with a lifetime or a failure rate as required by the application.

\section{TMR Length Sensors}

\section{Sensor Design}

TMR length sensors are designed to detect the periodic field of a magnetic scale (see Fig. 4). The sensors have the size of at least the length of a magnetic pole pair. It is possible to combine several length sensors for averaging over several pole pairs. This averaging concept reduces the position error caused by inhomogeneities of the magnetized poles.

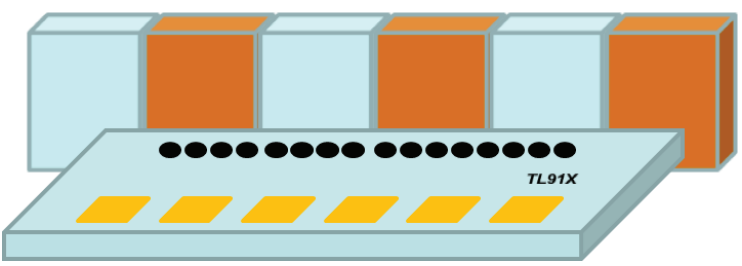

Figure 4: Arrangement of TMR length sensor and magnetic scale with periodic pole pairs. Black dots correspond to TMR junctions, yellow squares to bond pads.

Different pole pair lengths require different length sensors. Sensitec has designed TMR length sensors for pole pair structures of 0.5 $\mathrm{mm}, 1 \mathrm{~mm}, 2 \mathrm{~mm}, 3 \mathrm{~mm}$ and $5 \mathrm{~mm}$ (TL911TL915). The sensors perform in saturation mode, meaning that the magnetic field of the pole pairs saturates the magnetization of the sensing layer in the TMR layer stack.

\section{Performance}

As expected, the TMR length sensors provide signal amplitudes which are an order of magnitude larger compared to AMR length sensors. Therefore preamplifiers can be simplified or even be omitted.

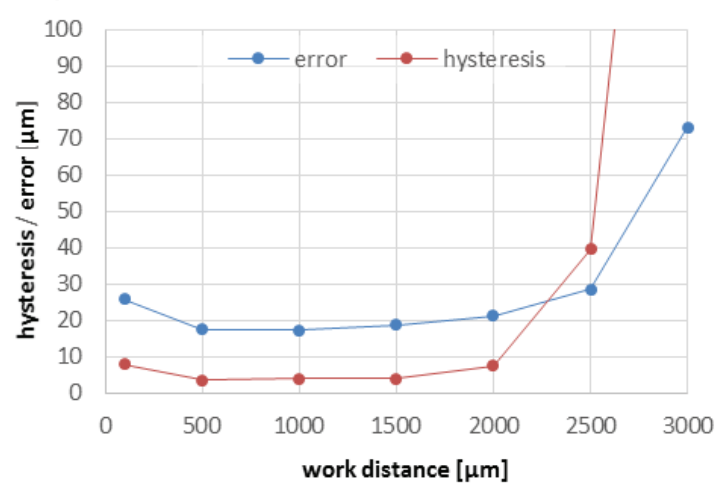

Figure 5: Absolute position error and hysteresis of TL915 (5 mm pole pair) in dependence of the airgap.
Position error was evaluated as a function of the airgap between sensor and magnetic pole structure. As can be seen in Fig. 5 the position error is in the range of $20 \mu \mathrm{m}$ for the $5 \mathrm{~mm}$ sensor for an air gap between 1-3 $\mathrm{mm}$. Hysteresis is very low in the range of only $5 \mu \mathrm{m}$. In general the absolute position error is approximately $0.5 \%$ and the hysteresis smaller $0.1 \%$ of the pole pair length.

\section{Lifetime Stability}

TMR sensors have been stressed in a HTOL test (high temperature operating life test). The conditions of the test have been $\mathrm{T}=150^{\circ} \mathrm{C}, \mathrm{B}=$ $30 \mathrm{mT}, \mathrm{t}=1000 \mathrm{~h}$ and $\mathrm{Vcc}=5.5 \mathrm{~V}$. The applied magnetic field in the oven was oriented perpendicular to the pinning direction, thus putting maximum stress onto the reference layer. Fig. 6 shows the result. Offset drift is smaller than $400 \mu \mathrm{V} / \mathrm{V}$. This absolute number is a factor of 10 larger compared to AMR sensors, but it should be related to the amplitude which is more than a factor of 10 larger compared to AMR. Therefore it can be concluded that TMR and AMR perform similar in lifetime tests.

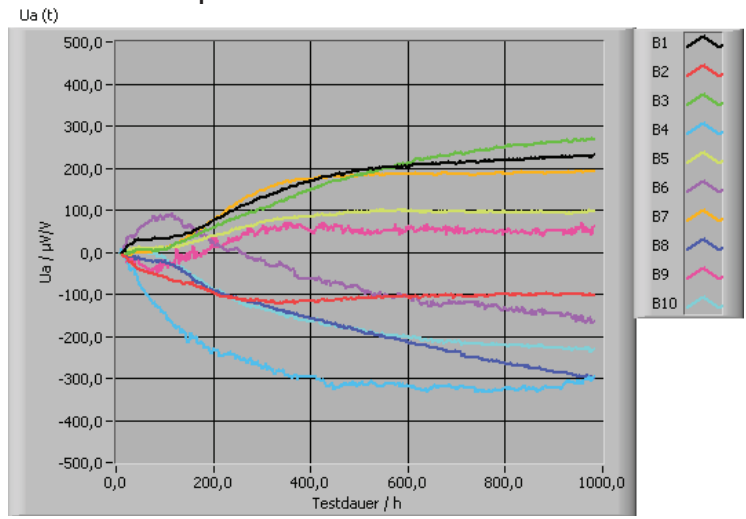

Figure 6: Offset drift during HTOL test of TL915. 


\section{TMR Angle Sensor}

An angle sensor for end of shaft application is under development by Sensitec, using TMR elements. This sensor consists of two Wheatstone bridges (sine and cosine), measuring signals corresponding to the two orthogonal components of the applied magnetic field vector. Each Wheatstone bridge incorporates at least two different pinning angles of the reference layer. Pinning angles of sine and cosine are rotated by $90^{\circ}$ [see Fig. 7].
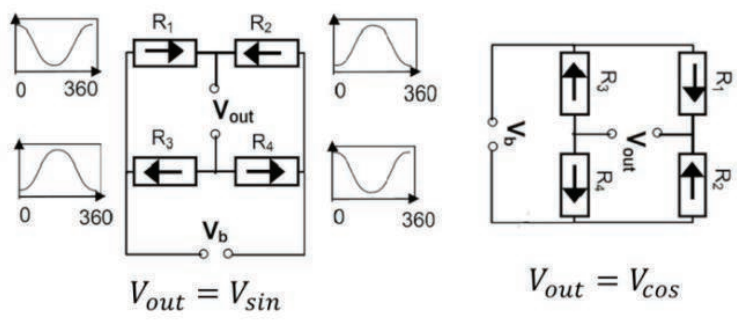

Figure 7: Basic design of a TMR angle sensor. Black arrows indicate the pinning directions in different parts of the Wheatstone Bridges.

The stability of the reference layer is given by exchange coupling and therefore limited against strong external fields. Applying rotating fields > $50 \mathrm{mT}$ yield in small oscillations of the magnetization direction of the reference layer and reduce the quality of the sine and cosine output signals. To extend the measurement range to magnetic fields $>100 \mathrm{mT}$, a concept of higher harmonic cancellation must be applied. In one of these concepts [15] the pinning angles for each resistor R1 - R4 are split into two pinning angles. The $\mathrm{n}$-th harmonic will be cancelled if the angle $\theta$ between the two pinning angles follows the equation:

$$
\theta=180^{\circ} / n
$$

With this filter concept eight pinning angles are already required for one angle sensor.

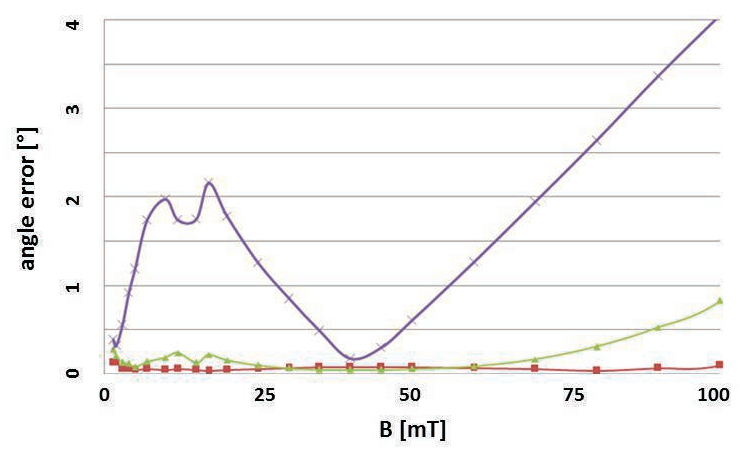

Figure 8: Simulation results of filter concepts. The purple line refers to the standard design shown in Fig. 7. The green line results applying one harmonic filter, the red line corresponds to an approach with two harmonic filters.
The impact of harmonic filters has been investigated by simulation before chip design. Fig. 8 demonstrates that angle errors below $0.2^{\circ}$ over a large field range can be realized by means of two filters. Consequently a large number of different pinning angles are needed. It is evident that a method of manufacturing multiple pinning angles in one chip must be available.

\section{Sensor Performance}

TMR angle sensors have been fabricated and characterized. Basic characteristics are: signal amplitude is in the range of $170 \mathrm{mV} / \mathrm{N}$, bridge offset is smaller than $2 \mathrm{mV} / \mathrm{V}$, orthogonality error between sine and cosine is $<2^{\circ}$ and amplitude synchronism is better than $1 \%$. Temperature coefficient of the amplitude is $-0.15 \% / \mathrm{K}$.

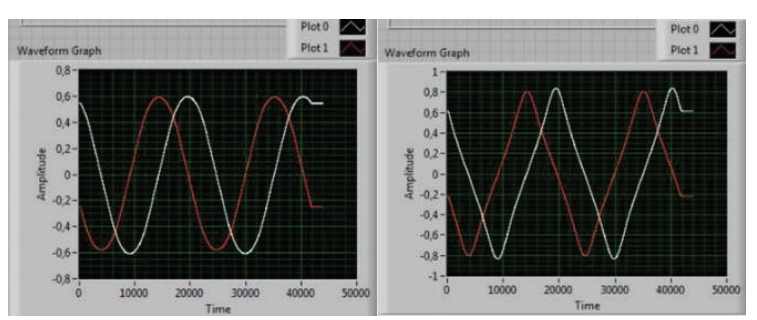

Figure 9: Sine and cosine output of TMR angle sensor at 90mT for design using harmonic filters (left) and design resigning any filtering concept.

Highest concern was given to the angle accuracy and the effect of the filtering concept. As can be seen in Fig. 9 sinusoidal curves can only be observed when applying harmonic filters.

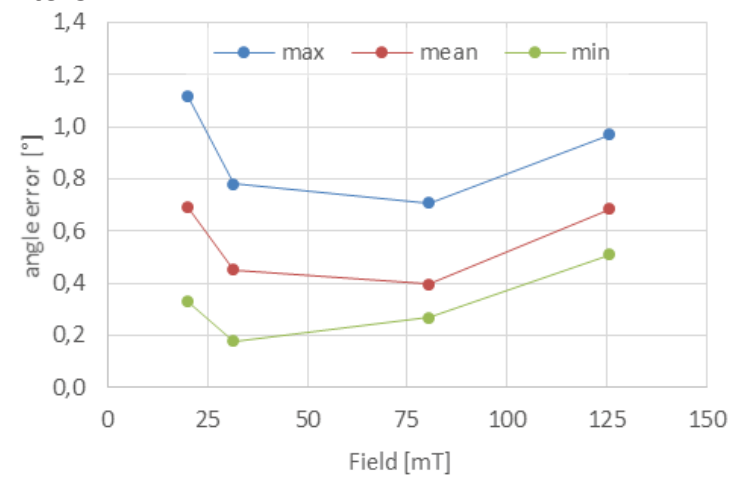

Figure 10: Angle error for TA901. Offset and amplitude are corrected but not the orthogonality error.

Typical absolute angle accuracy is $0.4^{\circ}$ in the range of 30-120 mT (see Fig. 10). Offset and amplitude are corrected in these analysis but not the phase error (orthogonality error), which is dominant in the low field range.

\section{Summary and Outlook}

TMR sensors are shown to be attractive in nearly all areas of magnetic sensors. They have 
proven to provide reliable data at high accuracy and significantly improved output signal compared to AMR and GMR sensors. Nevertheless the output amplitude can be further increased in the future. $300 \mathrm{mV} / \mathrm{V}$ seem to be realistic.

\section{References}

[1] See e.g. http://www.sensitec.com/upload/SENSITEC/ PDF Downloads/Datenblatt/Sensitec AA745A D SE 03.pdf

[2] See e.g. http://www.canonanelva.co.jp/english/info/ pdf/20131204EN.pdf

[3] J. Paul, R. Lehndorff, C. Duret, Ressourceneffizienz am Beispiel magnetoresistiver Sensoren, Proceedings of MST Kongress 2011 in Dresden, 293-296, 2011

[4] C. Duret, J. Paul, A. Gerken, R. Slatter, B. Negulescu, M. Hehn, Energieeffiziente, miniaturisierte magnetoresistive Sensoren für die Raddrehzah/sensorik und weitere automobile Anwendungen, Proceedings zur Konferenz Automotive meets Electronics AmE2011 in Dortmund, 2011

[5] R. Guerrero, M. Pannetier-Lecoeur, C. Fermon, S. Cardoso, R. Ferreira, and P. P. Freitas, Low frequency noise in arrays of magnetic tunnel junctions connected in series and parallel with built-in voltage, Appl. Phys. Lett. 105, 113922, 2009

[6] E. R. Novak, Ryan Stearrett, and K. M. Haughey, Overview of noise and its impact on the performance of modern magnetoresistive sensors, Proceedings zum 11. Symposium Magnetoresistive Sensors and Magnetic Systems, Wetzlar 2011

[7] F.A. Cardoso, L.S. Rosado, F. Franco, R. Ferreira, E. Paz, S.F. Cardoso, P.M. Ramos, M. Piedade, P.J.P. Freitas, Improved Magnetic Tunnel Junctions Design for the Detection of Superficial Defects by Eddy Currents Testing, IEEE Trans Magn. Vol 50-11 \# 6201304, 2014

[8] See e.g. http://www.singulus.de/uploads/tx pspublications/ Timaris 211114 01.pdf

[9] D.C. Worledge and P.L. Trouilloud, Appl. Phys. Lett. 83, 84 (2003)

[10] Kin P. Cheung, Plasma charging damage, Springer Verlag, p. 14, 2000

[11] J. Das, R. Degraeve, P. Roussel, G. Groeseneken, G. Borghs, J. De Boeck, Area scaling and voltage dependence of time-tobreakdown in magnetic tunnel junctions, J. Appl. Phys. 91, 7712, 2002

[12] H. Kim, J. Sok, B.K. Cho, J.R. Rhee, W. Parkand, T. Kim, Dielectric characteristics of magnetic tunnel junctions using amorphous CoNbZr layers, J. of the Korean Physical Society, 46, N. 6, 1425, 2005
[13] T. Min et al., A study of write margin of spin torque transfer magnetic random access memory technology, IEEE Tran. Magn. 46, 2322, 2010

[14] C. Yoshida, M. Kurasawa, Y. Min Lee, K. Tsunoda, M. Aoki, and Y. Sugiyama, A study of dielectric breakdown mechanism in $\mathrm{CoFeB} / \mathrm{MgO} / \mathrm{CoFeB}$ magnetic tunnel junction, Proceedings Reliability Physics Symposium (IRPS), IEEE International Conferences, 2009

[15] TDK patent application EP2 455720 A1

\section{Acknowledgement}

A. Conca and B. Leven acknowledge the support by the state of Rhineland-Palatinate (MBWWK and MWKEL) and by the European Regional Development Fund (ERDF) in the frame of the Spintronic Technology Platform (STeP) 